\title{
COMPARISON OF PERCUTANEOUS CROSS VERSES PARALLEL K WIRE FIXATION IN SUPRACONDYLAR FRACTURES OF THE HUMERUS IN CHILDREN.
}

\footnotetext{
1. FCPS Orthopedics

Senior Registrar Orthopedics SIMS.

2. FCPS Orthopedics

Senior Registrar Orthopedics SIMS.

3. MS Orthopedics

Medical Officer Orthopedics General Hospital, Lahore.

4. MBBS

Medical Officer

Services Hospital Lahore.

Correspondence Address:

Dr. Farhan Majeed

Senior Registrar Orthopedics SIMS.

farhanmajeed_85@hotmail.com

Article received on:

14/01/2019

Accepted for publication:

$14 / 06 / 2019$
}

\begin{abstract}
Farhan Majeed ${ }^{1}$, Mudasser Saddique ${ }^{2}$, Hafiz Nasir ${ }^{3}$, Ahmad Shams ${ }^{4}$
\end{abstract}
\begin{abstract}
Objectives: The aim of this study is to summarise and compare the radiological and functional results of two ways of fixation (cross and parallel closed $\mathrm{K}$ wires) of supracondylar fractures in children. Study Design: Randomized controlled trial. Setting: Department of Orthopaedics Surgery, Services Hospital, Lahore. Period: $1^{\text {st }}$ January 2018 to $31^{\text {st }}$ June 2018. Material \& Methods: We included 180 patients (90 in each group). Results: The mean age was $6.45 \pm 2.34$ years with $115(63.9 \%)$ male and $65(36.1 \%)$ female. Among the children who underwent fixation with cross k-wires, ulnar nerve injury was seen in $2(2.2 \%)$ cases and none were seen in the other group post operatively. Group $\mathrm{A}$ attained higher union rate at last follow up. $4(4.4 \%)$ cases in Cross K-wires and $19(21.1 \%)$ in two lateral k-wires gave outstanding outcome. In a nutshell, 60 in group A and 45 in group $B$ showed excellent outcomes based on Flynn's criteria, p-value $<0.05$. Conclusion: According to Flynn's criteria, closed percutaneous cross K-wire fixation of supracondylar fracture of humerus is an effective management option in terms of finer functional results as compared to Parallel k-wires. Although, the rate of radiological union is higher in cross k-wire fixation, there are $2.2 \%$ chances of ulnar nerve injury.
\end{abstract}

Key words: $\quad$ Close Reduction, Humerus, K-wire Fixation, Percutaneous Pinning, Parallel and Cross Wires, Supracondylar Fracture.

Article Citation: Majeed F, Saddique M, Nasir H, Shams A. Comparison of percutaneous cross verses parallel $\mathrm{k}$ wire fixation in supracondylar fractures of the humerus in children. Professional Med J 2020; 27(3):476-480.

DOI: 10.29309/TPMJ/2020.27.3.3091

\section{INTRODUCTION}

Supracondylar fractures are common in children and are associated with significant morbidity. ${ }^{1}$

Supracondylar fractures of the humerus represent $50-70 \%$ of all elbow fracture in children in the first decade of life. Current method of treatment of this fracture is based on Gartland classification. ${ }^{2}$

Out of the common complications associated with supracondylar fractures, some complications like mal-union, ischemic contracture and neurovascular damage are worrisome. ${ }^{3}$

Closed manipulation and percutaneous pinning of displaced supracondylar humeral fractures is commonly performed in the UK using two crossed pins or two lateral pins. Accurate reduction and stable fixation is key to the successful surgical treatment of these fractures, but this is often hampered by soft tissue swelling and gross fracture instability. ${ }^{4}$

Closed reduction and percutaneous Kirschner wire (k-wire )fixation is the preferred method of treatment in Gartland type 3 supracondylar humerus fractures in children. ${ }^{3}$

Lateral percutaneous pinning technique, of displaced Supracondylar fractures of the humerus offers a viable alternative to the crossed pinning group as it offers the same stability without the incipient risk of iatrogenic ulnar nerve injury. ${ }^{5,6}$

Closed reduction and percutaneous K-wire fixation is the standard method of managing displaced extension type supracondylar humerus fractures. Many investigators have used two crossed pins: one introduced medially and one laterally., 5

One trial found that excellent functional outcome $\left(<5^{\circ}\right.$ motion loss $)$ was observed in $81.8 \%$ 
cases with cross K-wire and $73.9 \%$ with parallel K-wires fixation $(p>0.05)$. But Baumann's angle was $73.09 \pm 4.888^{\circ}$ with closed reduction with cross K-wire and $76.05 \pm 3.240^{\circ}$ with parallel K-wire fixation $(p<0.05)$ for management of supracondylar humeral fracture in children. ${ }^{8}$

Rationale of this study is to compare the outcome of closed reduction and percutaneous cross versus parallel K-wires fixation in supracondylar humeral fracture in children. Literature reported that parallel technique is more effective in the management of supracondylar humeral fracture in children. But insignificant results have been observed. $^{8}$

Moreover, not much work has been done in this regard and local evidence also lacks. So there is a need to find the evidence to assess the best method for management of supracondylar humeral fracture in children. So, we want to conduct this study to get evidence regarding more effective and successful method for management of supracondylar humeral fracture in children. So that we may be able to implement the results of this study in local setting and apply more successful method in future.

\section{METHODOLOGY}

In this study 180 children were selected from the Accident \& Emergency and Orthopaedic Outpatient Department of Services Hospital Lahore from the duration of $1^{\text {st }}$ January 2018 to $31^{\text {st }}$ June 2018. Children with supracondylar fracture of humerus of either gender were selected, and those of ages above 16 years (the age of skeletal maturity) were excluded.

Patients presenting with complications of the fracture at the time of presentation were excluded from the study. After taking an informed consent from the guardians, name, age and gender were stated in statistics. Total patients were divided into two groups ( $A$ and $B$ ). The fractures of the children in group $A$, were reduced by closed technique under $\mathrm{C}$ Arm control with wire in cross manner, whereas the fractures in group $B$ were managed by parallel wires. Every patient of both categories was given a padded dressing and back slab was applied.

The patients were kept under observation for 12-24 hours and were discharged the next day after their full evaluation of neurovascular status. The patients were called for follow up at 1,2, 4, 6 and 12 weeks during which complete history, Physical examination and radiographs were recorded, Recording of Range of motion using Flynn's criteria (Table 1) for loss of motion and loss of carrying angle, was used. Splints were taken off after 4 weeks of surgery, followed by rehabilitation.

\section{RESULTS}

The mean age was $6.45 \pm 2.34$ years with $115(63.9 \%)$ male and 65(36.1\%) female. Among the children who underwent fixation with cross k-wires, ulnar nerve injury was seen in 2(2.2\%) cases and none were seen in the other group post operatively.

Group A attained higher union rate at last follow up. 4(4.4\%) cases in Cross K-wires and 19(21.1\%) in two lateral k-wires gave outstanding outcome. In a nutshell, 60 in group A and 45 in group B showed excellent outcomes based on Flynn's criteria (Table 2), p-value $<0.05$.

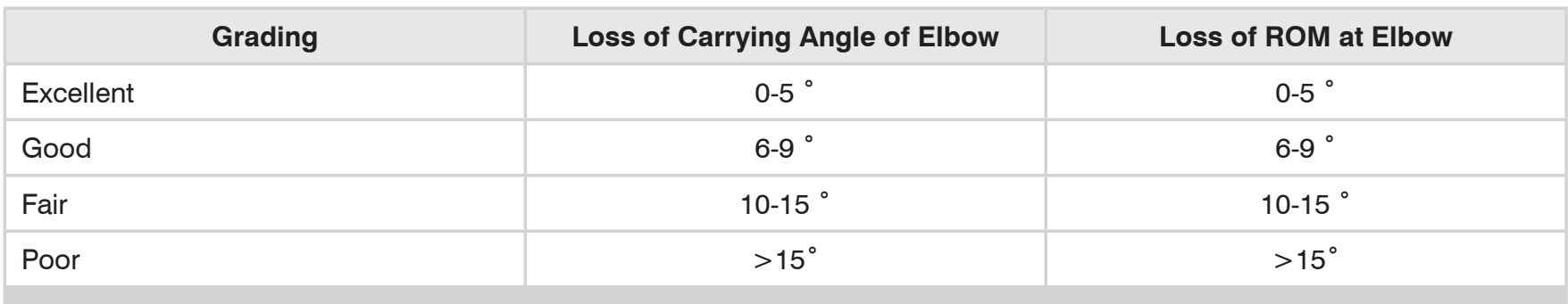

Table-I. Flynn criteria ROM = Range of Motion 


\begin{tabular}{|l|c|c|c|c|}
\hline \multicolumn{1}{|c|}{ Grading } & $\begin{array}{c}\text { Loss of Carrying } \\
\text { Angle of Elbow }\end{array}$ & $\begin{array}{c}\text { Loss of ROM at } \\
\text { Elbow }\end{array}$ & Group A & Group B \\
\hline Excellent & $0-5^{\circ}$ & $0-5^{\circ}$ & 60 & 45 \\
\hline Good & $6-9^{\circ}$ & $6-9^{\circ}$ & 26 & 32 \\
\hline Fair & $10-15^{\circ}$ & $10-15^{\circ}$ & 4 & 13 \\
\hline Poor & $>15^{\circ}$ & $>15^{\circ}$ & 0 & 0 \\
\hline
\end{tabular}
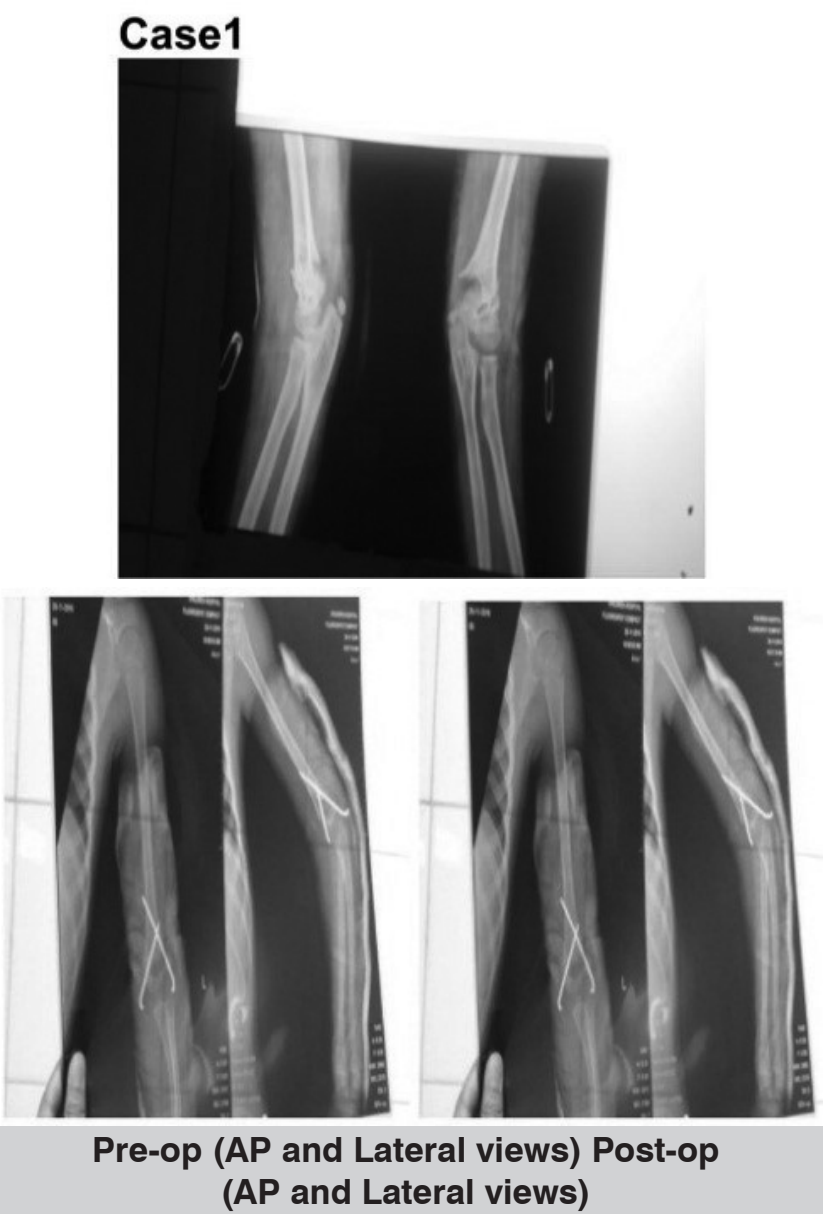

\section{DISCUSSION}

Being the most common elbow fractures $\left(1 / 3^{\text {rd }}\right.$ of all fractures that occur in children ${ }^{9}$ ) supracondylar fractures usually occur in 2-8 years of age, mostly affecting the left limb. These are more common in boys. ${ }^{10}$ In one study, mean age of patients was $6.41 \pm 2.37$ with boys. ${ }^{11}$

Mean age in cross k-wires and two lateral k-wires was $6.24 \pm 2.94$ and $6.61 \pm 1.24$ years respectively with no prominent difference $p$ value $>0.05$.

These are mostly caused by low energy trauma

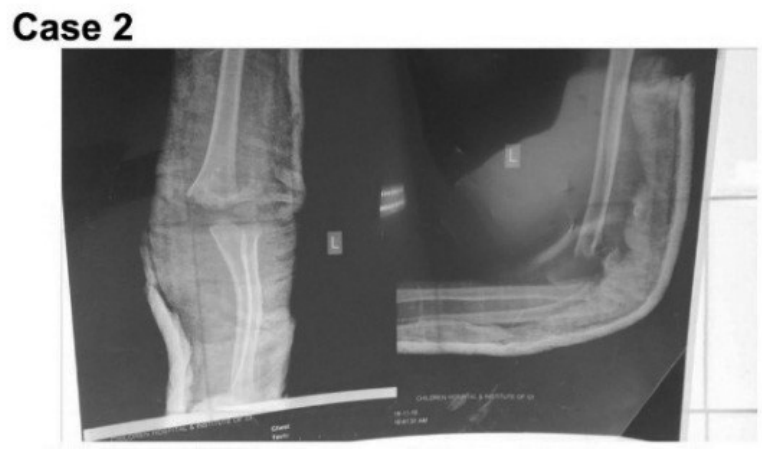

\section{Pre-op (AP and Lateral views)}

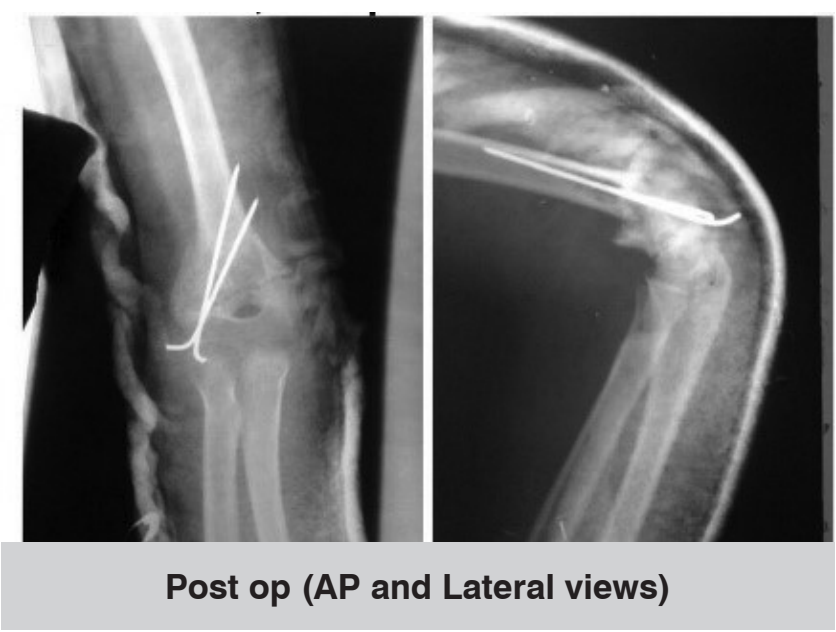

and are categorized on the basis of injury pattern, which are extension and flexion types respectively. Injury caused by extension occurs due to fall on the palm of hand with hyperextended elbow (most common), while direct fall onto fixed elbow results in flexion type of these injuries. ${ }^{12}$ Out of these traumatic injuries, displaced ones are consequently known for neurological and vascular issues. Surgery is recommended in such cases, for casting and immobilizing the elbow often results in neurovascular complications, however the type of procedure is still debatable. 
Various treatment options are available, e.g. different configurations of k-wires like two parallel, divergent or cross k-wires (medial and lateral) and POP case for un-displaced fractures. Each has its own merits and demerits, for instance, laterally placed divergent pins protect the ulnar nerve and provide equal stability like cross wire. Overall, divergent pattern is more stable than parallel one. ${ }^{13}$

The procedure of Closed reduction and internal fixation is although performed with patient lying in supine position, one study however, reported a new technique of reducing a Displaced Supracondylar humeral fracture by traction and gradual extension in the prone position. ${ }^{14}$

On the other hand, typical current management for displaced fracture is closed reduction and percutaneous $\mathrm{K}$ wire fixation, which has consistently given excellent results reported by various authors. ${ }^{15}$ However, consensus has still not been made on the arrangement, whether cross or two parallel lateral wires are better in terms of stability and iatrogenic ulnar nerve damage which formed the rationale of this study. Ideally medial and lateral pins engage medial and lateral columns at injury site, whereas, lateral pins stabilize lateral and central columns.

According to a study in 2011, medial and lateral cross k-wires fixation group gave $72 \%$ excellent and $28 \%$ good results, while similar outcome were found in 2 lateral k-wires group. ${ }^{16}$

The cross wires have been demonstrated in biomechanical studies and clinical trials to be more stable configuration than others (Braner et al 2007). Conversely, lateral pins have been used by all except to avoid ulnar nerve injury, however it is considered less stable biochemically.

\section{CONCLUSION}

According to Flynn's criteria, closed percutaneous cross K-wire fixation of supracondylar fracture of humerus is an effective management option in terms of finer functional results as compared to Parallel k-wires. Although, the rate of radiological union is higher in cross k-wire fixation, there are
$2.2 \%$ chances of ulnar nerve injury.

Copyright $\subset 14$ June, 2019.

\section{REFERENCES}

1. Khwaja MK, Khan WS, Ray P, Park DH. A retrospective study comparing crossed and lateral wire configurations in paediatric supracondylar fractures. The open orthopaedics journal 2017; 11:432.

2. Sahu R. Percutaneous lateral Kirschner wire fixation in pediatric supracondylar fractures of humerus. Journal of Orthopedics, Traumatology and Rehabilitation [Original Article] 2013 January 1, 2013; 6(1):78-83.

3. Prashant K, Lakhotia D, Bhattacharyya TD, Mahanta AK, Ravoof A. A comparative study of two percutaneous pinning techniques (lateral vs medial-lateral) for Gartland type III pediatric supracondylar fracture of the humerus. Journal of Orthopaedics and Traumatology 2016; 17(3):223-9.

4. Dekker A, Krijnen P, Schipper I. Results of crossed versus lateral entry K-wire fixation of displaced pediatric supracondylar humeral fractures: A systematic review and meta-analysis. Injury 2016; 47(11):2391-8.

5. Gopinathan NR, Sajid M, Sudesh P, Behera P. Outcome analysis of lateral pinning for displaced supracondylar fractures in children using three kirschner wires in parallel and divergent configuration. Indian journal of orthopaedics 2018 Sep-Oct; 52(5):554-60.

6. Sahu RL. Percutaneous K-wire fixation in paediatric Supracondylar fractures of humerus: A retrospective study. Niger Med J 2013; 54(5):329-34.

7. Sapkota K, Wahegaonkar K, Ranjeet N, Thapa U, Onta P. Comparison of cross pinning versus lateral three pins in type three supracondylar fracture of distal humerus in children. Asian Journal of Medical Sciences 2019 03/01; 10:58-61.

8. Gopinathan N, Sajid M, Sudesh P, Behera P. Outcome analysis of lateral pinning for displaced supracondylar fractures in children using three kirschner wires in parallel and divergent configuration. Indian Journal of Orthopaedics [Symposium - Pediatric Trauma] 2018 September 1, 2018; 52(5):554-60.

9. Omid R, Choi P, Skaggs D. Current concepts review - supracondylar humeral fractures in children. Bone Joint Surg Am 2008; 90: 1121-32.

10. Babal, J.C. Mehlman, C. T. \& Klein, G. 2010. Nerve injuries associated with paediatric supracondylar humeral fractures; A meta-analysis. Journal of Pediatric Orthopedics, 30, 253-263. 
11. Sapkota, K. \& Shrestha, B. 2014. Study of supracondylar fracture of distal humerus in children and its management with lateral $k$-wire fixation. Nepal J Med Sci, 3, 38-43.

12. Pavone V, Riccioli M, Testa G, Lucenti L, De Cristo C, Condorelli G, Avondo S, Sessa G. Surgical treatment of displaced supracondylar pediatric humerus fractures: comparison of two pinning techniques. Journal of Functional Morphology and Kinesiology. 2016 Mar;1(1):39-47.

13. Lee, S. S., Mahar, A. T., Miesen, D. \& Newton, P. O. 2002. Displaced pediatric supracondylar humerus fractures: Biomechanical analysis of percutaneous pinning techniques. J Peads Orthop, 22, 440-443.
14. Belhan, O., Karakurt, L., Ozdemir, H., Yilmaz, E., Kaya, M., Serin, E., et al. 2009. Dynamics of the ulnar nerve after percutaneous pinning of supracondylar humeral fractures in children. Journal of Pediatric Orthopaedics B, 18, 29-33.

15. Anwar W, Rahman N, Khan MA, Idrees M. Radiological Evaluation of The Stability of Crossed-Pins Versus Lateral-Pins Fixation in Displaced Supracondylar Fractures of Humerus. Journal of Surgery Pakistan (International). 2013 Jul;18:3.

16. Kao HK, Lee WC, Yang WE, Chang $\mathrm{CH}$. Treatment of displaced flexion-type pediatric supracondylar humeral fractures in the prone position. Journal of Orthopaedic Surgery. 2017 Jan 18;25(1):2309499016684412.

\begin{tabular}{|}
\hline \multicolumn{3}{|c|}{ AUTHORSHIP AND CONTRIBUTION DECLARATION } \\
\hline Sr. \# & \multicolumn{1}{|c|}{ Author(s) Full Name } & Contribution to the paper & Author(s) Signature \\
\hline 1 & Farhan Majeed & Surgeries + Data Collection. \\
\hline 2 & Mudasser Saddique & Surgeries + Data collection. \\
\hline 3 & Hafiz Nasir & Data collection. \\
\hline 4 & Ahmad Shams & Data collection. \\
\hline
\end{tabular}

